\title{
Magnetic resonance imaging of bone marrow in oncology, Part 2
}

\author{
Sinchun Hwang • David M. Panicek
}

Received: 19 February 2007 / Revised: 5 March 2007 / Accepted: 7 March 2007 / Published online: 11 May 2007

(C) ISS 2007

\begin{abstract}
Magnetic resonance imaging plays an integral role in the detection and characterization of marrow lesions, planning for biopsy or surgery, and post-treatment followup. To evaluate findings in bone marrow on MR imaging, it is essential to understand the normal composition and distribution of bone marrow and the changes in marrow that occur with age, as well as the basis for the MR signals from marrow and the factors that affect those signals; these points have been reviewed and illustrated in part 1 of this two-part article. Part 2 will emphasize the practical application of MR imaging to facilitate differentiation of normal marrow, tumor, and treatment-related marrow changes in oncology patients, and will review complementary MR techniques under development.
\end{abstract}

Keywords Magnetic resonance imaging · Marrow .

Metastases $\cdot$ Tumor $\cdot$ Sarcoma $\cdot$ Treatment effects

\section{Tumor in marrow}

Malignant tumors in marrow can arise from the myeloid elements of marrow (resulting in leukemia, lymphoma,

Part 1 of this article can be found at http://dx.doi.org/10.1007/s00256007-0309-3.

\section{S. Hwang $\cdot$ D. M. Panicek $(\bowtie)$}

Department of Radiology,

Memorial Sloan-Kettering Cancer Center,

1275 York Avenue,

New York, NY 10021, USA

e-mail: panicekd@mskcc.org.

S. Hwang · D. M. Panicek

Weill Medical College of Cornell University,

New York, NY 10021, USA myeloma), from the mesenchymal elements of marrow (resulting in primary sarcoma of bone), or from hematogenous metastases to marrow. Tumor can also reach the marrow by direct extension from a location outside the bone. Tumors occur more commonly in red marrow because of its richer vascularity than that of yellow marrow.

\section{Lymphoproliferative diseases}

In leukemia, the infiltration of bone marrow typically is diffuse, resulting in a diffuse decrease in marrow signal intensity on T1-weighted images. However, very early in the disease, tumor cells may not completely replace fat cells in marrow and thus the resultant marrow signals can be normal on T1- and T2-weighted spin echo (SE) images. In multiple myeloma, the amount of marrow fat can even increase initially because the tumor cells produce biochemical factors that inhibit hematopoiesis [1]. When more extensive, lymphoproliferative tumors in marrow are low in signal intensity on T1-weighted SE images and high in signal intensity on $\mathrm{T} 2$-weighted $\mathrm{SE}$ images.

Bone marrow necrosis (BMN) is an unusual entity that has been reported to occur in close association with malignancy, particularly acute lymphocytic leukemia. BMN is characterized by a loss of myeloid tissue and marrow fat without trabecular destruction, unlike avascular necrosis [2]. Despite its relative rarity $(0.3-0.4 \%$ of marrow biopsies) [3, 4], it is important to recognize the close association between BMN and malignancy. In a study of 240 patients with BMN, Janssens et al. [2] reported that $91 \%$ of patients were found to have malignancy, with acute lymphocytic leukemia representing $18 \%$ of the cases. BMN may be found at the time of cancer diagnosis or later in its course. MR imaging findings of BMN are similar to those 
of avascular necrosis (AVN), although BMN tends to be more diffuse, involving the axial skeleton without vertebral collapse; AVN, in contrast, involves periarticular locations and may progress to vertebral fracture [5].

In multiple myeloma, the infiltrative pattern on MRI is variable, ranging from normal to focal or diffuse (Fig. 1). Although diffuse disease may mimic the MRI appearance of extensive red marrow reconversion (Fig. 1b,c), the signal intensity on fat-suppressed T2-weighted SE images is generally considerably higher than that of muscle (Fig. 1d). Due to the lack of associated osteoblastic activity, nuclear bone scans and radiographs are often normal in multiple myeloma, making MRI the most sensitive imaging method for lesion detection. Studies have shown that MRI findings correlate with treatment response and survival [1, 6, 7]. Independent studies by Moulopoulos et al. [6] and Vande Berg et al. [7] suggested that patients with stage I disease who had normal-appearing bone marrow on MRI had a longer progression-free interval, and patients with bone marrow involvement evident on MRI had more rapid progression of the disease. In a study of 80 patients with stage III multiple myeloma, Lecouvet et al. [8] showed that a good or partial response to chemotherapy (determined by a $50-75 \%$ decrease in serum or urine myeloma protein) was more common in patients with normal-appearing marrow on MRI than in patients with an abnormal-appearing marrow (89\% versus $68 \%$, respectively). A diffusely abnormal pattern of bone marrow also correlated with more marked alterations in other clinical parameters of disease activity such as serum calcium, t32 microglobulin and hemoglobin levels, indicating more severe disease than found in patients with a focally abnormal MRI pattern of bone marrow [8]. Stabler et al. [1] evaluated five types of infiltration patterns and confirmed the prognostic value of MRI in staging of, and in planning treatment for, multiple myeloma. They also reported a correlation between the pattern of infiltration and the degree of contrast enhancement: A greater than $40 \%$ increase in signal intensity compared with baseline after contrast administration correlated with diffuse tumor infiltration of marrow.
Fig. 1 Multiple myeloma: multifocal and diffuse patterns. a Coronal T1-weighted spin echo (SE) MR image shows innumerable tiny foci of low signal intensity on a background of fatty marrow. b Coronal T1weighted SE MR image of a different patient shows diffuse, symmetric, low signal intensity in the marrow of proximal and mid femurs, mimicking the appearance of red marrow. c Coronal fat-suppressed T2-weighted MR image of the left hip of the same patient as in $\mathbf{b}$ reveals diffuse, markedly high signal throughout the marrow, indicating the presence of marrow disease rather than red marrow

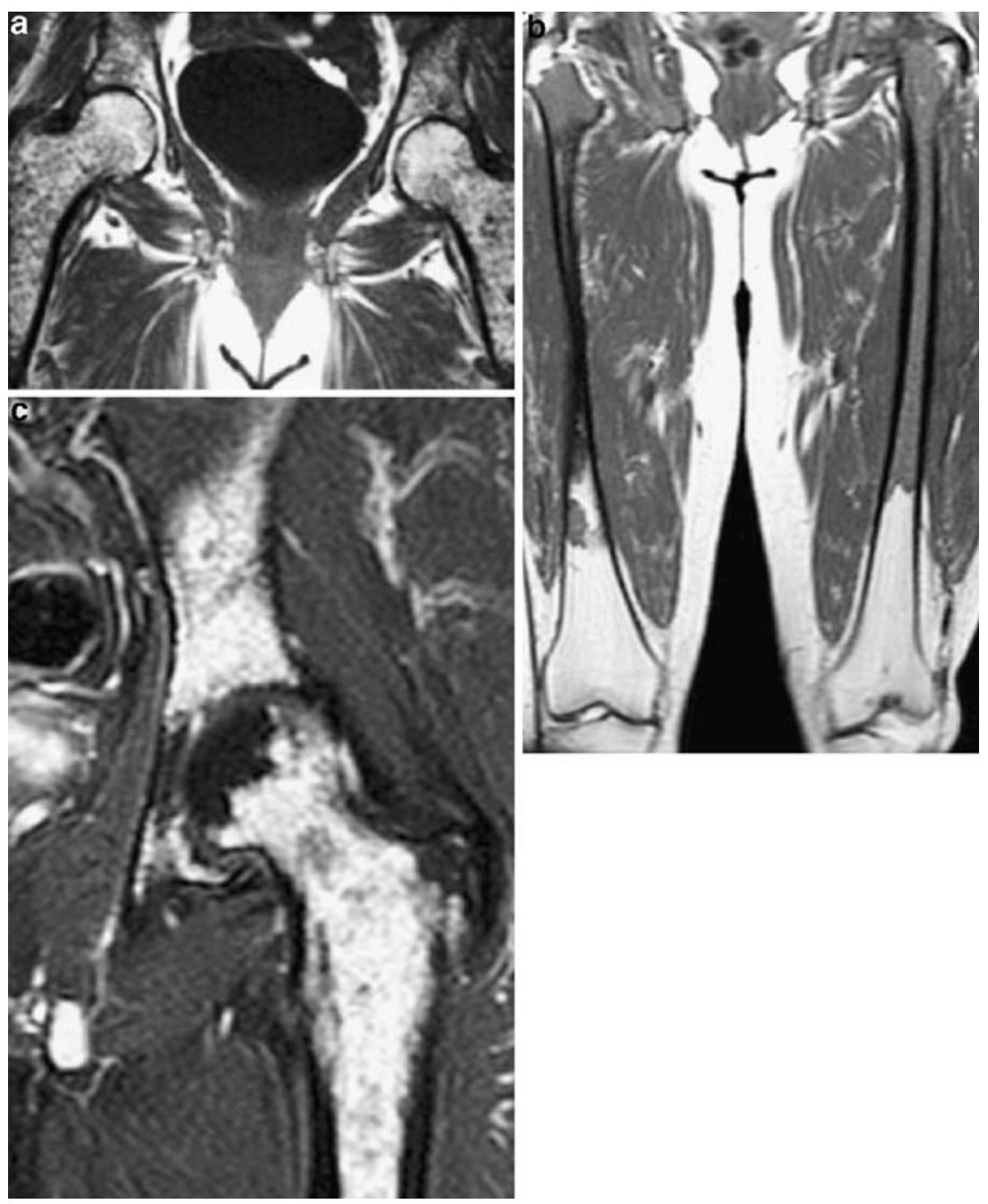


Fig. 2 Multifocal Hodgkin disease, with false-negative blind marrow biopsy from the posterior ilium. Axial a, b T1weighted and $\mathbf{c}, \mathbf{d}$ corresponding fat-suppressed T2-weighted SE MR images show focal tumor deposits in marrow of the right iliac wing and left acetabulum (long arrows). Note linear biopsy tract (short arrows) within normal-appearing marrow of the right posterior ilium; this biopsy yielded a false-negative marrow assessment
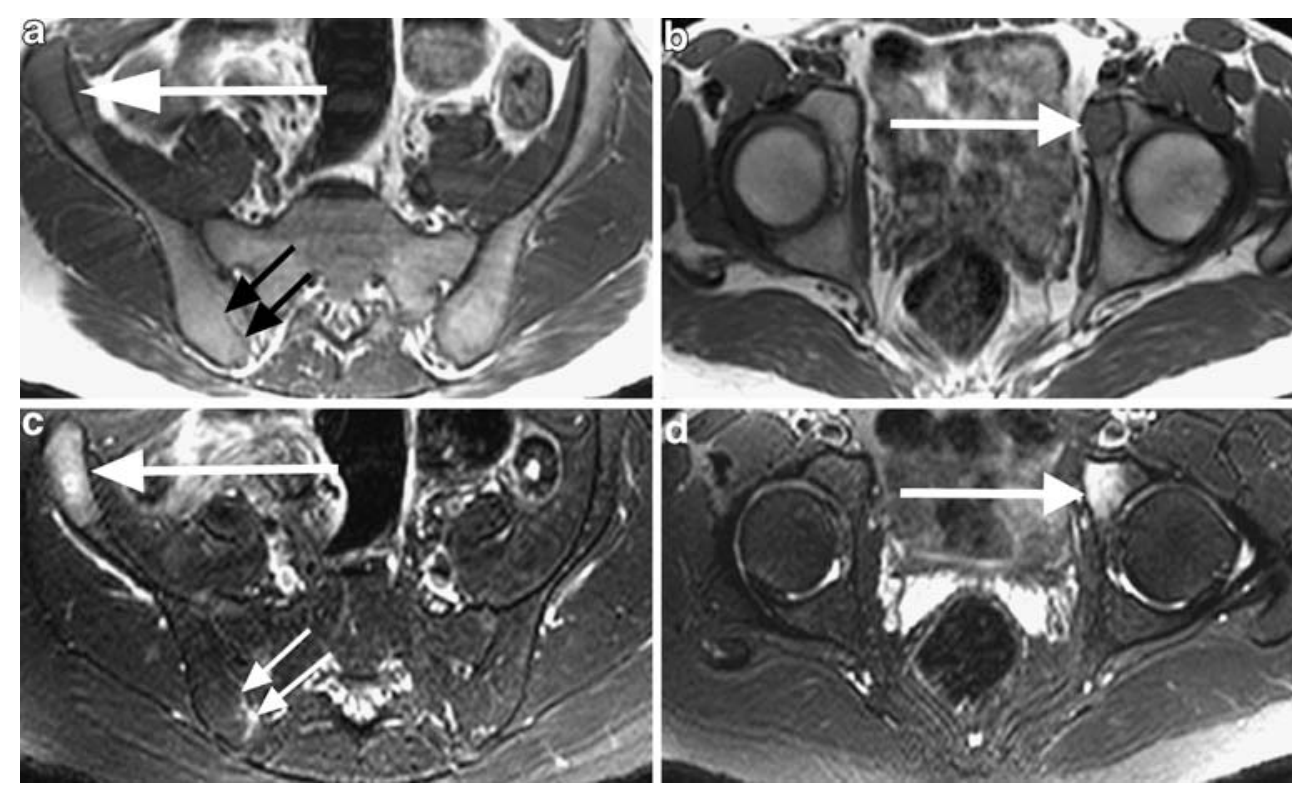

Lymphoma in bone marrow can also be focal or diffuse. The ability to detect focal lymphoma has substantial consequences, as the presence or absence of bone marrow involvement is used to assess patient prognosis and select the specific type(s) of therapy [9]. In non-Hodgkin lymphoma, marrow involvement is more frequent than in Hodgkin disease (25-45\% of patients in the former, versus $5-15 \%$ in the latter) [10]. Because marrow involvement in lymphoma is often multifocal but not diffuse, a blind biopsy of marrow can be falsely negative due to sampling error (Fig. 2). This phenomenon was demonstrated in studies of bilateral biopsies in which positive findings were present only unilaterally in $10-40 \%$ of patients with marrow involvement by lymphoma and other diseases [11, 12]. MR imaging can be used to more fully assess marrow involvement in lymphoma, and to specifically identify appropriate potential biopsy sites. Also, dynamic contrastenhanced MRI may be useful to assess treatment response, as a decrease in degree of contrast enhancement has been shown to correlate with a good response or remission [13].

\section{Metastases}

Bone marrow metastases are more commonly seen in red marrow but can be present anywhere that marrow exists. The MRI appearances of metastases vary depending on the balance between the tumor and the response of the host bone; metastases can be osteolytic, osteoblastic or mixed. When osteolytic, metastases are usually well circumscribed with low signal intensity on T1-weighted images and moderately high signal intensity on T2-weighted images. In osteoblastic metastases, such as those from breast and prostate carcinomas, metastases may show low signal intensity on both T1and $\mathrm{T} 2$-weighted images because relatively less tumor is present between the sclerotic trabeculae (which themselves lack MR signal).

A rim of high signal surrounding a lesion ("halo sign") or diffuse signal hyperintensity of a lesion on T2-weighted images have been reported as useful indicators in the diagnosis of metastasis (sensitivity $75 \%$; specificity $99.5 \%$; Fig. 3) [14]. This halo sign can potentially be useful in
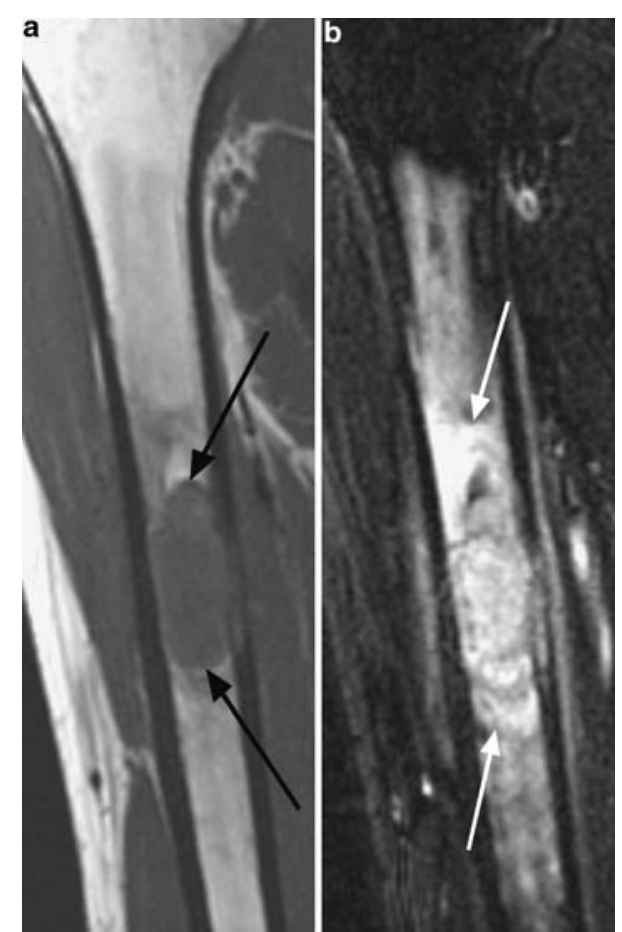

Fig. 3 Halo sign in thyroid metastasis. a Coronal T1-weighted SE MR image shows well-defined lesion of homogeneous low signal intensity (arrows) in humeral shaft. b Coronal fat-suppressed T2weighted MR image demonstrates extensive, poorly defined high signal surrounding lesion (arrows), consistent with edema 
evaluating small blastic metastases that might otherwise be mistaken for bone islands (Fig. 4). In contrast, the bull'seye sign, when present, has been shown to be a useful tool in determining that a region of altered marrow signal is not due to metastasis, with a specificity of $99.5 \%$ [14]. The sign consists of one or more foci of high signal (due to fat) present in marrow within a region of low signal intensity on T1-weighted images.

Some tumors, such as small cell carcinoma, grow between trabeculae within marrow without causing gross trabecular or cortical destruction; similar growth may occur in the earliest stages of tumor extension within marrow. MRI is superior to radiographs and bone scan in detecting such intertrabecular tumor deposits (Fig. 5); its sensitivity $(94.6 \%)$ is much higher than that of radiography $(5.8 \%)$ or bone scan (3.3\%) [15]. In a study by Taoka et al. [16] for detection of spinal metastases using MRI, corresponding bone scans were positive in $71 \%$ of transcortical metastases, only $34 \%$ of subcortical metastases, and $0 \%$ of totally intramedullary metastases. MRI clearly shows more metastases than bone scans do, as many metastases do not have cortical involvement.

Of note, one subtype of soft tissue sarcoma, myxoid liposarcoma, is well known to produce asymptomatic marrow metastases that are not evident on radiographs or bone scan [17]. Such tumor deposits in marrow are readily demonstrated on MRI (Fig. 6).

\section{Post-treatment changes}

During and after treatment for tumors in marrow, the affected marrow undergoes substantial changes in its chemical and cellular composition [18-20]. Because MRI is sensitive and noninvasive in detecting changes in the composition of marrow, MRI findings have been extensively investigated to assess for early and late treatmentrelated changes and for the presence of residual tumor.
Fig. 4 Halo sign in tiny metastasis from prostate cancer. a Axial T1-weighted SE MR image shows tiny, ill-defined lesion with low signal intensity (arrow) in the right ischium. b Axial fat-suppressed T2-weighted MR image shows low signal intensity within the lesion (long arrow), and peripheral rim with high signal intensity ("halo sign"; short arrows). c Axial contrast-enhanced fat-suppressed T1weighted MR image reveals mild enhancement of the rim (halo) around the lesion (arrows). d Lesion is sclerotic on CT (arrow), and could have been misdiagnosed as a bone island
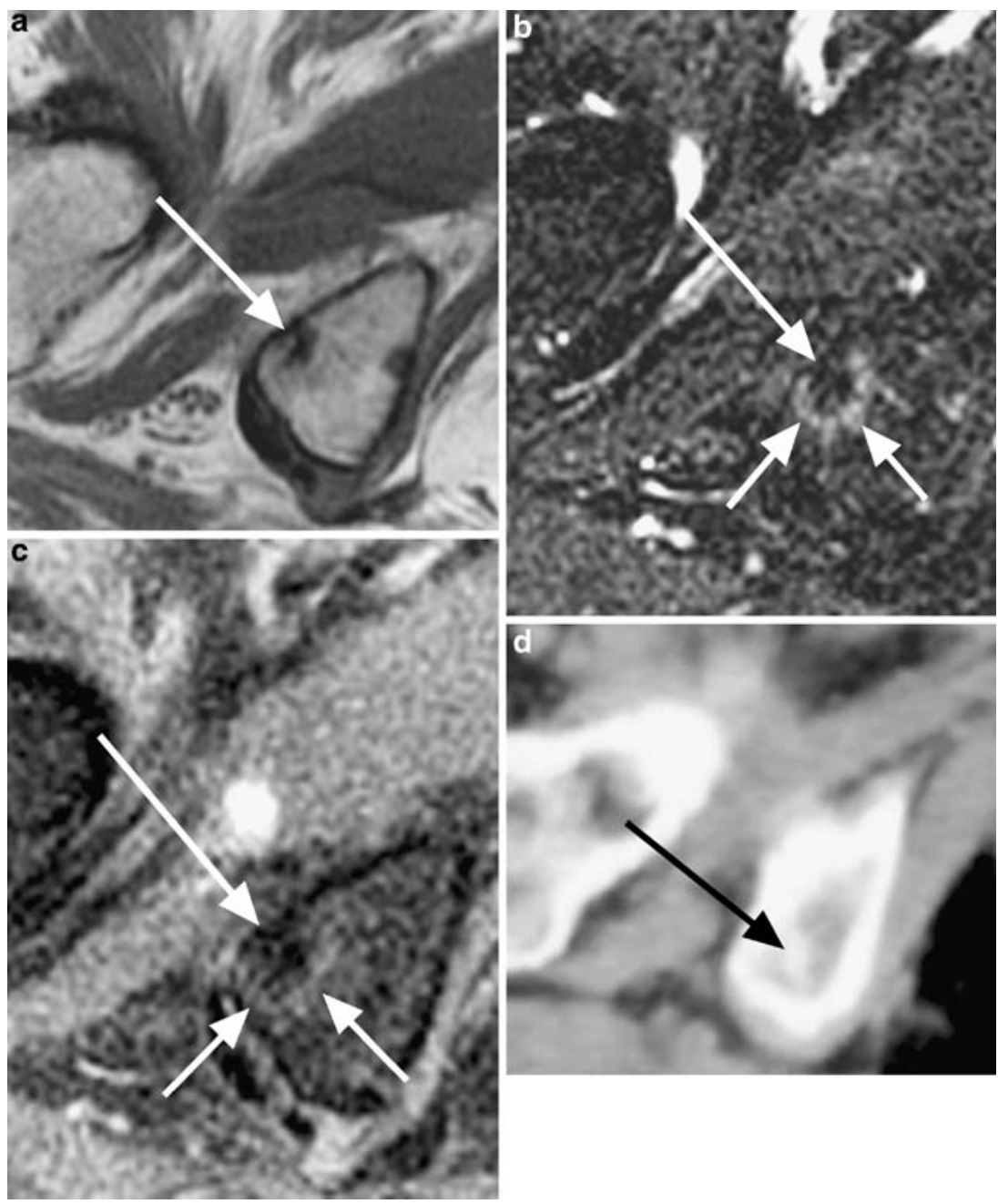

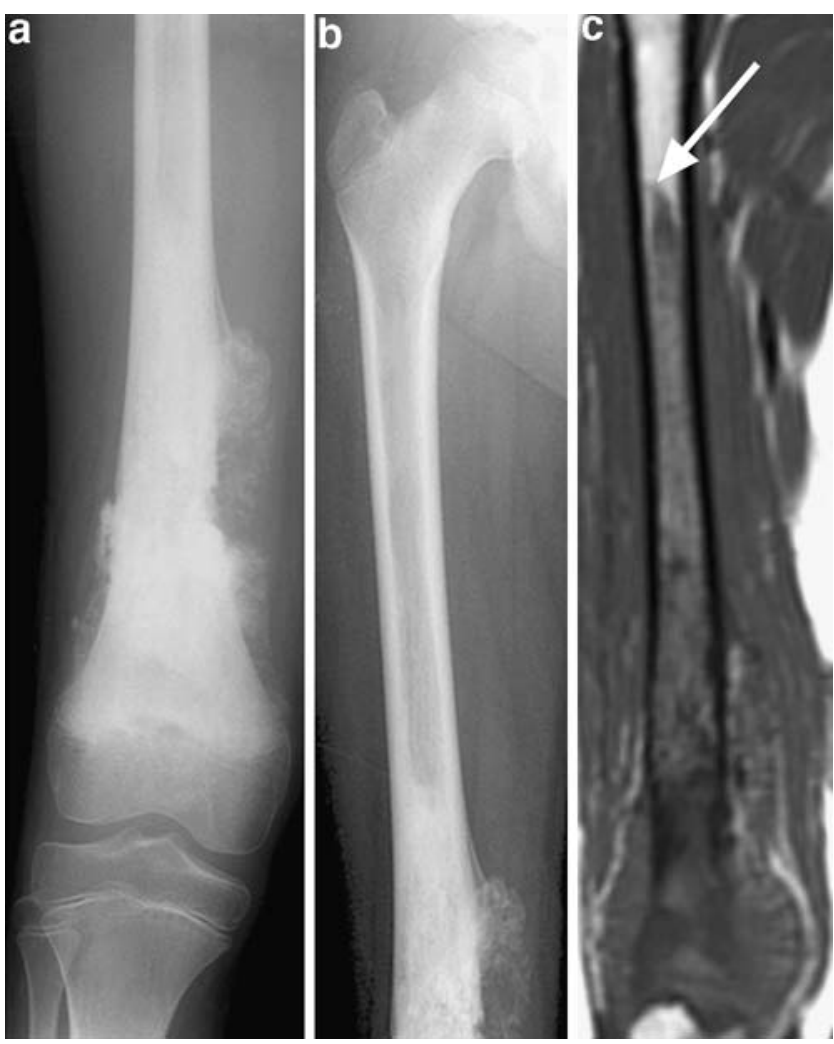

Fig. 5 Extent of marrow involvement in osteosarcoma. a, b Anteroposterior radiographs show a classic osteosarcoma in the distal femur; the exact proximal extent of the tumor is unclear. c Coronal T1-weighted SE MR image readily shows the proximal extent of abnormal marrow (arrow), which extends into the proximal diaphysis

Changes in marrow signal due to radiation therapy

On both MRI and histopathologic evaluation, acute and chronic changes induced by radiation treatment (RT) have been well described [21-24]. In the acute phase, cellular depletion, marrow edema, vascular congestion, and hemorrhage ensue during the first hours and days. Within 1-2 weeks, the observed increase in MR signal intensity in T2-weighted and STIR images is thought to represent a reduction in cellularity and an increase in fat and marrow edema $[22,24]$. An increase in MR signal on STIR images can be observed as early as 8 days after initiation of RT [22].

In the chronic phase of post-RT change, a high T1weighted MR signal of irradiated marrow compatible with yellow marrow has been observed 3-7 weeks after the initiation of RT (Fig. 7) [22]. In the spine, a band pattern of peripheral, intermediate $\mathrm{T} 1$ signal intensity has also been noted, and is thought to represent red marrow surrounding a central fatty deposit [24].

The temporal evolution of MR signal changes in an individual patient depends upon several factors. In a study of 48 patients who underwent RT, Sacks et al. [19] reported that the two most important factors affecting the ability of marrow to regenerate are patient age and radiation dose. They found that younger patients had superior marrow recovery, with full marrow regeneration occurring in patients under 18 years of age, independent of radiation dose. Also, the capacity to regenerate marrow treated with a dose greater than 40 Gy was less than in marrow treated with a lower dose. Most regeneration occurred within 12 months of RT [19].

Although lesser and smaller, changes in MR signal are also observed in bone marrow located outside the radiation portal of the spine; the etiology of such changes remains unclear [22, 25]. Contrast enhancement of marrow both inside and outside the radiation portal decreases significantly with time; it has been speculated that this decrease in enhancement represents reductions in cellularity and vascularity, and an increase in fatty marrow in response to RT [25].

Radiation treatment-induced complications in the marrow include osteonecrosis, AVN, fractures, and radiationinduced neoplasm, and depend on factors such as radiation dose, patient age, size of the radiation field, and beam energy [26]. Cell death and devascularization of bone occur after doses exceeding 5,000 cGy [26]; these events impair hematopoietic regeneration and normal bone formation. Osteonecrosis/AVN is localized within the radiation portal and usually is diagnosed years after RT; typical locations include the mandible, clavicle, humeral head, rib, and femur. On MRI, the presence of high signal intensity inside a low-intensity peripheral rim on T2weighted images (the "double line" sign) is specific for the diagnosis of AVN [27].

Fractures of irradiated bones, such as sacral insufficiency fractures or collapsed vertebrae, can occur within a few months of RT and may present diagnostic challenges by mimicking osseous metastases. Correct diagnosis is imperative to avoid unnecessary biopsies and to guide proper clinical management. Particularly in patients with osteoporosis (such as postmenopausal women), fracture risk after RT is substantially increased. Abe et al. [28] reported a $34 \%$ prevalence of sacral insufficiency fractures in a retrospective study of 80 patients who underwent bone scanning; of note, all insufficiency fractures were identified in postmenopausal women. Multiple fractures may develop within 24 months [29]. The incidence of RT-induced sacral insufficiency fractures seen on MRI is $20-80 \%$ [29, 30]. The fracture lines usually parallel the sacroiliac joints and show ill-defined margins in all sequences. MRI may demonstrate the fractures earlier than CT by revealing surrounding edema, before fracture lines become visible on CT (Fig. 8).

Radiation treatment-induced bone tumors can be benign or malignant. The prevalence of (benign) osteochondroma after RT in patients with unfused physes has been reported to be $12 \%$ at 8 years - a prevalence incidence higher than in 
Fig. 6 Occult marrow metastasis myxoid liposarcoma. a Coronal T1-weighted SE MR image shows large lesion with low signal intensity (arrow) in the marrow of the right proximal femur, representing metastasis from a previously resected myxoid liposarcoma of the soft tissues of the right distal thigh. Abnormal signal in the adjacent soft tissues of the lateral hip (asterisk) reflects biopsy changes. b Marrow metastasis in the right proximal femur is radiographically occult, and was not visible on the bone scan (not shown). (Case courtesy of Cristina Antonescu, MD, Memorial Sloan-Kettering Cancer Center, New York, NY, USA)
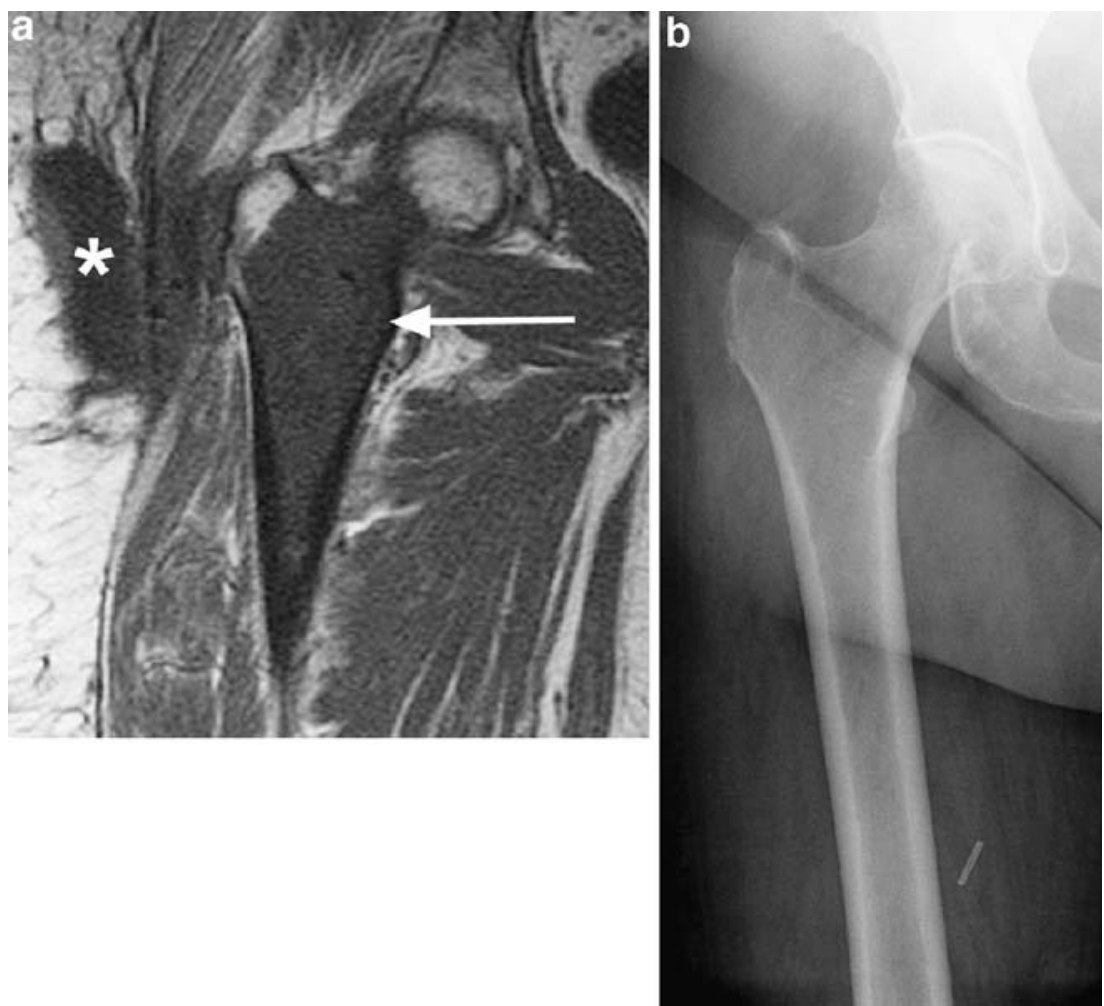

spontaneous osteochondroma (1\%) [31]. An RT-induced sarcoma of bone is rather rare, constituting less than $1.5 \%$ of all bone sarcomas [32]. By definition, the sarcoma must be histologically distinct from the original treated lesion, and occur within the irradiated field, typically after a long latent period [33]. Although the lowest dose required to induce a sarcoma is not established, studies have suggested that there is an association between the radiation dose and subsequent risk of sarcoma. In a study of 16 patients after

Fig. 7 Post-radiation marrow changes in the thoracic spine. Sagittal T1-weighted SE MR image shows diffuse fatty marrow throughout the portion of the thoracic spine that had been included within the radiation therapy port; the most cephalad and caudad vertebrae included in the port are indicated by asterisks. Therapy was directed at spinal metastases (the largest located within the T8 vertebral body)

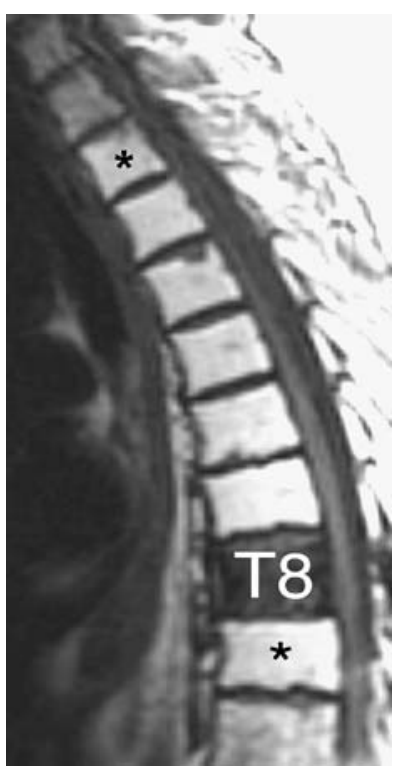

RT for Ewing sarcoma, Kuttesch et al. [34] reported that no secondary sarcoma was observed if the radiation dose was less than $48 \mathrm{~Gy}$; excess risk of a secondary sarcoma was associated with radiation doses greater than $60 \mathrm{~Gy}$, confirming the findings of previous studies. High-grade osteosarcoma, fibrosarcoma and malignant fibrous histiocytoma are the most common histologic types of radiationinduced sarcomas. The mean latency period ranges from 14 to 17 years (although some have occurred as soon as 3 years after RT), and the tumors are usually radioresistant [32, 35]. RT-induced sarcomas were initially considered to have a grave prognosis compared with primary bone sarcoma, but subsequent studies have shown that aggressive treatment with chemotherapy and surgical resection improves the prognosis of RT-induced sarcomas, resulting in more than a $60 \% 5$-year survival rate - approaching that of a primary bone sarcoma (50-80\% 5-year survival rate) $[33,35]$.

\section{Changes in marrow signal due to chemotherapy}

Upon initiation of chemotherapy, marrow signal becomes hypointense on T1-weighted images and hyperintense in fat-suppressed T2-weighted and STIR images, reflecting marrow congestion [36]. About 3-4 weeks into chemotherapy, reconversion from fatty marrow to hematopoietic marrow can occur, and is readily recognized as it usually occurs in the reverse sequence of marrow conversion, both bilaterally and symmetrically. After chemotherapy, depletion 

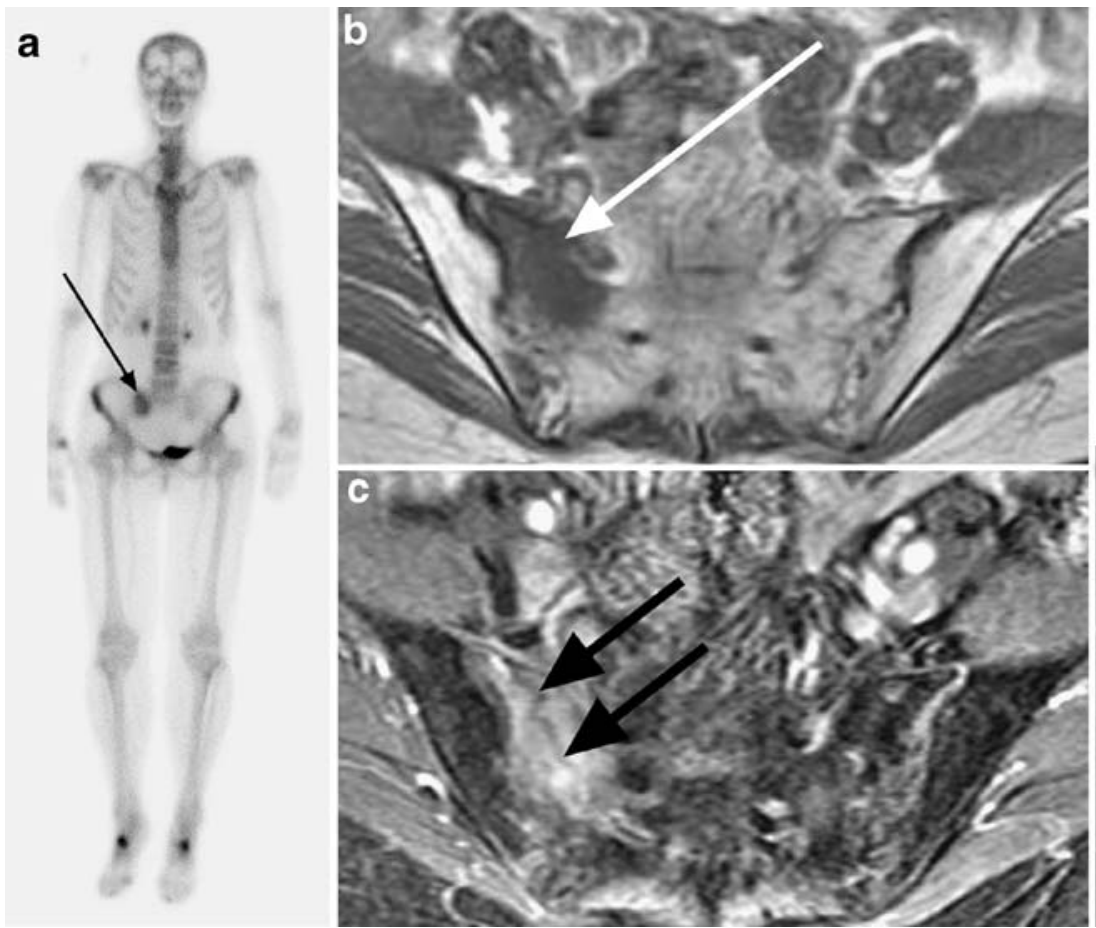

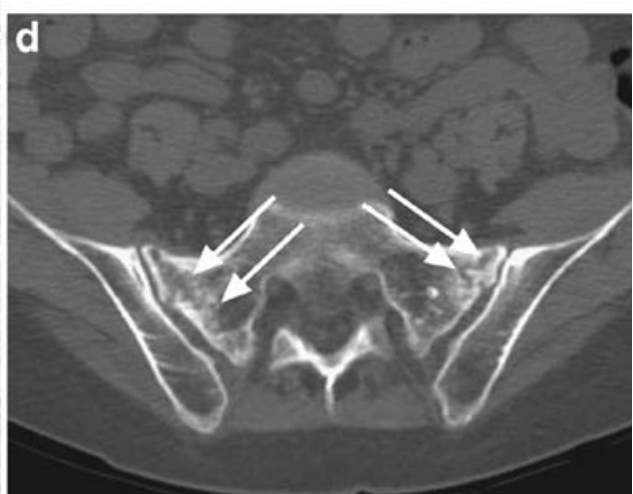

Fig. 8 Sacral insufficiency fracture in postmenopausal woman treated with radiation 2 years earlier for cervical cancer. a Bone scan (anterior view) shows intense focal uptake (arrow) in the right sacrum, initially interpreted as metastasis. b Axial T1-weighted SE MR image shows abnormal marrow signal in the right sacral ala (arrow), which could be consistent with metastasis. c Axial contrast-enhanced fat-suppressed
T1-weighted gradient echo MR image, however, shows linear low signal (arrows) within corresponding region, diagnostic of sacral insufficiency fracture. No fracture was visible on initial CT (not shown). d CT obtained 5 months later clearly demonstrates (now) bilateral sacral insufficiency fractures (arrows) of hematopoietic marrow may result in a predominantly fatty marrow $[18,36]$.

Several drugs used in oncology patients can cause various side effects in immature and mature skeletons, some of which can be misdiagnosed as tumors on MRI. Osteopenia, fractures, osteonecrosis, and AVN (Fig. 9) are well-known complications of prolonged administration of steroids in patients of all ages. In children, methotrexate can cause osteopenia, severe bone pain and metaphyseal insufficiency fracture (described as methotrexate osteopathy) [37]. In breast cancer treatment, both hormonal therapy with aromatase inhibitors in postmenopausal women and chemotherapy-induced ovarian failure in premenopausal women can cause significant loss of bone mass, increasing the risk of fractures [38].

Bisphosphonates (BP) are osteoclast inhibitors that can be used for the treatment of bone loss and bone pain in metastatic disease and multiple myeloma. Cases of osteonecrosis of the jaw in patients treated with BP have been recently and increasingly reported, strongly suggesting a causal link [39]. Osteonecrosis of the jaw is usually painful and preceded by a dental procedure in $60 \%$ of cases. Oversuppression of bone turnover and antiangiogenic effects of BP are considered to be contributing factors $[39,40]$.
The effects of granulocyte-colony stimulating factor (GCSF) in bone marrow are often encountered on MRI, as GCSF is increasingly used in conjunction with chemotherapy. G-CSF is an agent used to promote hematopoietic activity (specifically, stimulation of the granulocyte cell line) in order to decrease the incidence of chemotherapy-induced neutropenia. Multiple areas of red marrow develop in conjunction with increasing neutrophil counts. However, there is no apparent relationship between the number of treatment courses of G-CSF and the extent of red marrow reconversion (Fig. 10) [41]. The signal characteristics of the changes in marrow are similar to those of red marrow in both pediatric and adult patients [42, 43]. Although this drug-induced red marrow can simulate marrow disease on MRI, combined knowledge of the effects of G-CSF on marrow and the clinical status of individual patients can help to avoid misdiagnosing this treatment-related effect as an interval development of metastases [44]. For example, it would be highly unusual for multiple bone metastases to develop in a patient whose primary tumor was either unchanged or decreasing in size during chemotherapy; in such a case, it would be appropriate to report the new findings in the marrow as being consistent with the effects of marrow-stimulating therapy. 
Fig. 9 Marrow infarcts after chemotherapy for acute lymphoblastic leukemia. a Sagittal T1-weighted SE MR image shows typical serpiginous rims with low signal intensity surrounding regions of fatty marrow in distal femur and proximal tibia. b Sagittal fatsuppressed T2-weighted MR image shows a double-line sign (arrow), consisting of an inner band of high signal intensity and an outer band of dark signal

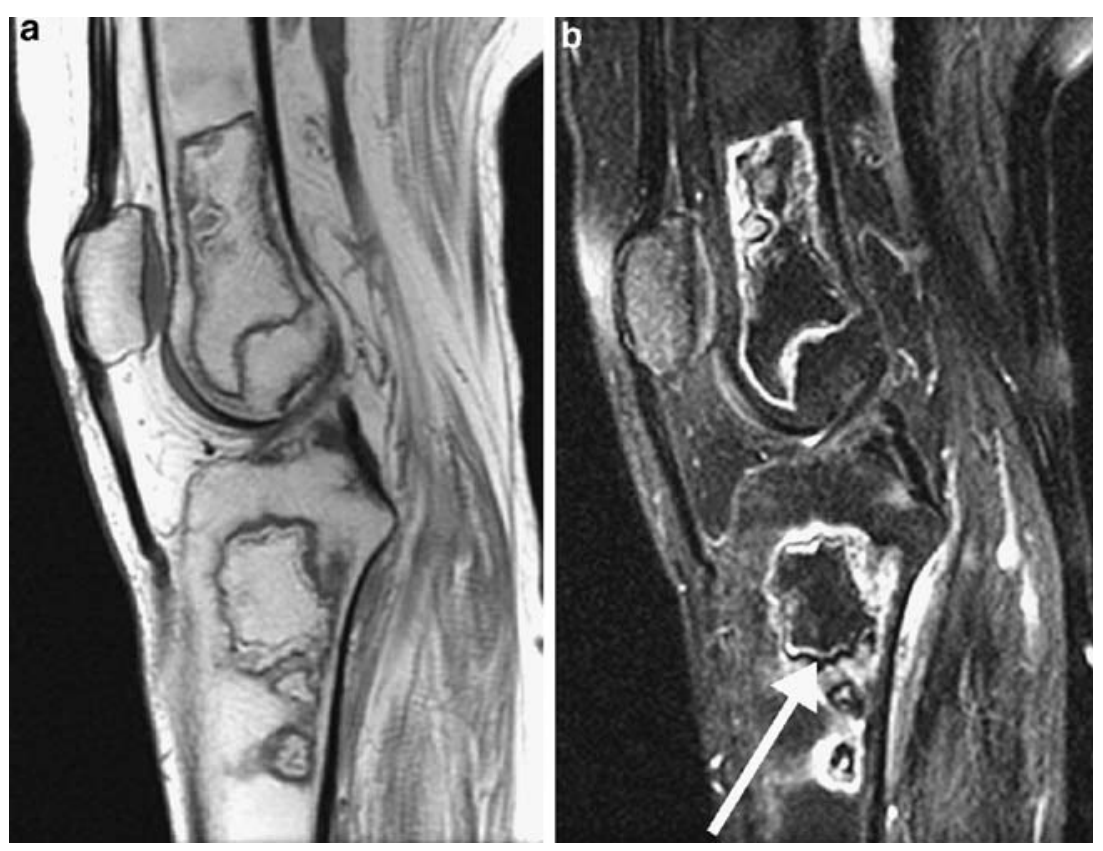

\section{Complementary MR techniques under development}

Although MRI is effective in diagnosing and monitoring malignant marrow diseases, it lacks specificity in distinguishing viable tumor from treatment-related changes (Fig. 11) [45, 46]. Hanna et al. [45] correlated MRI findings in a variety of disseminated marrow diseases with marrow biopsies at 21 sites and found that MRI was nondiscriminatory in differentiating post-treatment changes from malignant disease in all MR sequences obtained. Seven lesions were found to contain viable tumor at biopsy; the majority of T1-weighted, T2weighted, and STIR sequences showed abnormal signal in these lesions, and all post-contrast sequences showed lesional enhancement. However, among 14 lesions that did not contain viable tumor at biopsy, abnormal signal was present in the lesions on the majority of T1-weighted, T2weighted, and STIR sequences, and enhancement was present in more than half of the lesions on post-contrast sequences. These findings underscore the lack of specificity of signal changes on MRI for distinguishing viable tumor and post-treatment changes.

In order to increase the specificity and accuracy of MRI in the clinical setting, different MR techniques have been investigated, mainly focusing on the quantitative assessment of a variety of parameters. Techniques that have been evaluated include quantitative $\mathrm{T} 1$ relaxation time measurement, diffusion-weighted echo-planar imaging (EPI), chemical shift imaging, and dynamic contrast enhancement [47-53]. Although still in a relatively early stage of development, these techniques have shown the potential to complement conventional MRI. For example, in quantita- tive analyses of $\mathrm{T} 1$ relaxation time in leukemia by Vande Berg et al. [49], a decrease in T1 values in acute lymphocytic leukemia showed a correlation with therapy response (a mean 50\% decrease in $\mathrm{T} 1$ values in patients with a response, versus a mean $18.7 \%$ decrease in patients without a response), whereas $\mathrm{T} 1$ values in acute myeloid leukemia did not. However, in a study of quantitative MRI using T1 relaxation times, Lecouvet et al. [51] reported that MRI failed to detect leukemic marrow infiltration in $41 \%$ of patients with chronic lymphocytic leukemia.

In a study of lymphoma involving iliac bone marrow by Yasumoto et al. [47], the sensitivity and specificity of diffusion-weighted EPI imaging (77\% and 97\%, respectively) were statistically similar to those of fast SE STIR imaging ( $85 \%$ and $97 \%$ respectively). T1-weighted imaging had higher sensitivity (92\%) but lower specificity (77\%) than diffusion-weighted EPI [47]. The EPI technique is limited by poor spatial resolution and by susceptibility and chemical shift effects [52]. Quantitative chemical-shift imaging enables the quantitative assessment of fat content in bone marrow, thus allowing for noninvasive monitoring of temporal changes in marrow composition $[48,53]$. In a study of 11 patients with acute leukemia by Gerard et al. [48], sequential increases in fat fraction were correlated with the estimated fat fraction on biopsy, which reflects clinical remission; however, the baseline fat fraction and the increases in fat fraction were variable across individuals.

For more than a decade, a potential role for dynamic contrast-enhanced MRI has been explored in the assessment of the percentage of necrosis present within a bone tumor after treatment. This research is motivated by the finding that the percentage of necrosis following neo- 
Fig. 10 Marrow changes due to G-CSF treatment after chemotherapy for osteogenic sarcoma of proximal tibia. a Axial T1weighted SE MR image shows several small, ill-defined foci of slightly decreased signal intensity (arrows) relative to fatty marrow in ankle. b Axial fatsuppressed T2-weighted MR image shows corresponding high signal intensity within the foci (arrows). In this clinical scenario, these MR findings are compatible with islands of red marrow developing due to treatment with G-CSF, and should not be mistaken for metastases
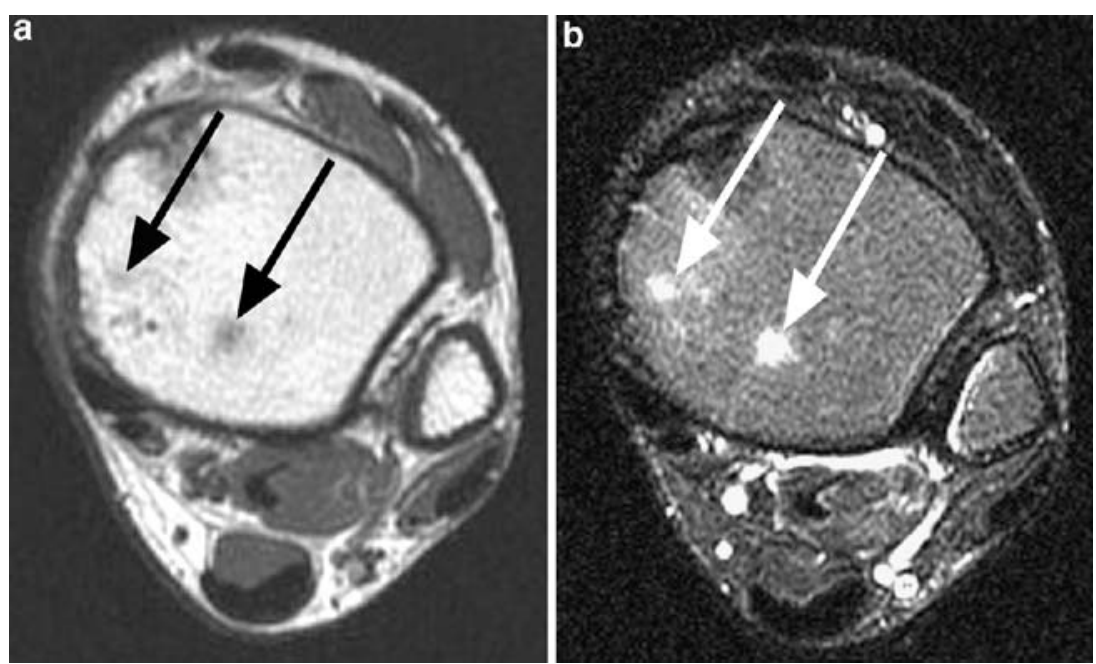

adjuvant chemotherapy portends patient prognosis in osteogenic sarcoma [54, 55]. In a study of 50 cases of osteogenic sarcoma and Ewing sarcoma by Dyke et al. [50], quantitative estimates of tumor necrosis obtained using dynamic contrast-enhanced MRI were shown to be strongly correlated with the percentage of necrosis determined by pathologic evaluation (using the Huvos grading system), particularly in making the clinically relevant distinction between those tumors that are less or more than $90 \%$ necrotic. These findings suggest that dynamic MRI could be valuable for noninvasively assessing treatment response during neoadjuvant therapy, potentially allowing a change to be made in that therapy for those patients whose response is poor. Currently, the software used for data analysis is developed at each institution that performs necrosis studies; until some such proprietary software is made commercially available, the utility of this technique will be limited.

Recent advances in MR technology have made wholebody MRI (WBMRI) possible in a single session. For example, the introduction of a rolling-table platform with integrated surface coils has eliminated previously time-consuming changes in position of both patient and coils, and sequences such as breath-hold gradient echo and whole-body turbo STIR have substantially shortened scan time-in some cases to a total scan time of less than 15 min-while also providing good spatial resolution [56-58]. Given its high sensitivity in the detection of
Fig. 11 Treatment effect versus residual viable osteosarcoma in proximal tibia after neoadjuvant chemotherapy. Coronal a fatsuppressed T2-weighted MR image and $\mathbf{b}$ corresponding gross pathology specimen show extensive abnormality within the marrow of the tibia. The amount of residual viable tumor cannot be assessed by visual inspection of the MR image or gross specimen. Histopathologic analysis using the Huvos method determined that $40 \%$ of the remaining lesion was necrotic, indicating a poor (grade I) chemotherapeutic response
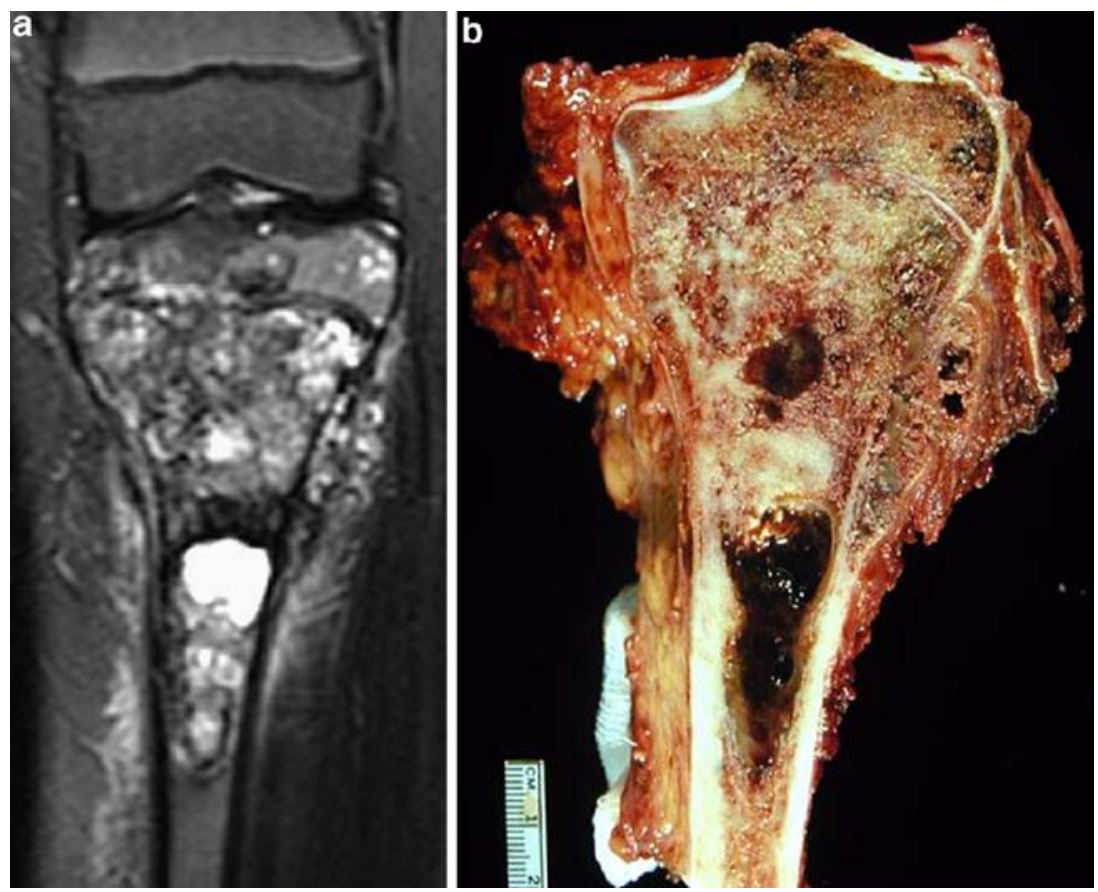
marrow lesions and excellent soft tissue contrast, WBMRI is under investigation as a screening and staging modality in oncology patients [56-61]. For example, WBMRI appears to be both more sensitive and specific than radiographic skeletal survey in assessing the extent of multiple myeloma, which is critical in determining the appropriate therapy $[58,59]$. In a study of 51 patients, Lauenstein et al. [60] compared WBMRI with CT, conventional dedicated MRI, and bone scanning; they found that WBMRI was more sensitive than those conventional modalities in detection of hepatic and osseous metastases. WBMRI and CT showed hepatic metastases in 18 and 16 patients, respectively, whereas WBMRI and bone scanning showed osseous metastases in 24 and 21 patients, respectively. They also found that WBMRI and conventional modalities possess specific advantages in different anatomic regions; for example, WBMRI detected more osseous metastases in the spine and pelvis, whereas bone scanning detected more metastases in the skull and ribs. In a study of 129 patients, Ghanem et al. [61] reported an $81 \%$ concordance between WBMRI and bone scanning; among 24 discordant cases, WBMRI detected osseous metastases in 15 cases that bone scanning did not. Given the ongoing development of faster and more efficient scanning techniques, the clinical utility of WBMRI is likely to increase in the future.

\section{Summary}

Magnetic resonance imaging is an excellent noninvasive modality for evaluating bone marrow and detecting marrow lesions, as it provides information at the level of cellular and chemical composition, in addition to gross morphologic data. Knowledge of normal marrow components and composition and their variation, as well as of factors that alter MR signal intensity, is important for optimal interpretation of MR images. The signal intensity, morphology, and location of marrow findings on MRI can be used to provide more accurate diagnoses, to guide treatment, and to follow therapy-related changes. Various MRI techniques are available to accentuate the different chemical and cellular compositions of normal marrow and marrow diseases. Although MRI is more sensitive than specific in detecting marrow changes, integrating all the clinical and radiologic data can result in more useful interpretations. Recognition of posttreatment effects, such as those associated with RT and granulocyte-colony-stimulating factors, are important for proper interpretation of MRI. In an attempt to overcome the current limitations of MRI, several newer techniques are under investigation that could increase the ability of
MRI to provide even more clinically relevant information about marrow in oncology patients.

\section{References}

1. Stabler A, Baur A, Bartl R, Munker R, Lamerz R, Reiser MF. Contrast enhancement and quantitative signal analysis in MR imaging of multiple myeloma: assessment of focal and diffuse growth patterns in marrow correlated with biopsies and survival rates. Am J Roentgenol 1996; 167: 1029-1036.

2. Janssens AM, Offner FC, Van Hove WZ. Bone marrow necrosis. Cancer 2000; 88: 1769-1780.

3. Pennaforte JL, Dufour M, Etienne JC, Schvartz H, Caulet T. Diagnostic problems of bone marrow necrosis. Apropos of a case disclosing acute leukosis. Arch Anat Cytol Pathol 1986; 34: 111-116.

4. Dunn P, Shih LY, Liaw SJ, Sun CF. Bone marrow necrosis in 38 adult cancer patients. J Formos Med Assoc 1993; 92: 1107-1110.

5. Tang YM, Jeavons S, Stuckey S, Middleton H, Gill D. MRI features of bone marrow necrosis. Am J Roentgenol 2007; 188: 509-514.

6. Moulopoulos LA, Dimopoulos MA, Smith TL, et al. Prognostic significance of magnetic resonance imaging in patients with asymptomatic multiple myeloma. J Clin Oncol 1995; 13: 251-256.

7. Vande Berg BC, Lecouvet FE, Michaux L, et al. Stage I multiple myeloma: value of MR imaging of the bone marrow in the determination of prognosis. Radiology 1996; 201: 243-246.

8. Lecouvet FE, Vande Berg BC, Michaux L, et al. Stage III multiple myeloma: clinical and prognostic value of spinal bone marrow MR imaging. Radiology 1998; 209: 653-660.

9. Varan A, Cila A, Büyükpamukçu, M. Prognostic importance of magnetic resonance imaging in bone marrow involvement of Hodgkin disease. Med Pediatr Oncol 1999; 32: 267-271.

10. Linden A, Zankovich R, Theissen P, Diehl V, Schicha H. Malignant lymphoma: bone marrow imaging versus biopsy. Radiology 1989; 173: 335-339.

11. Coller BS, Chabner BA, Gralnick HR. Frequencies and patterns of bone marrow involvement in non-Hodgkin lymphomas: observations on the value of bilateral biopsies. Am J Hematol 1977; 3: 105-119.

12. Brunning RD, Bloomfield CD, McKenna RW, Peterson L. Bilateral trephine bone marrow biopsies in lymphoma and other neoplastic diseases. Ann Intern Med 1975; 82: 365-366.

13. Rahmouni A, Montazel JL, Divine M, et al. Bone marrow with diffuse tumor infiltration in patients with lymphoproliferative diseases: dynamic gadolinium-enhanced MR imaging. Radiology 2003; 229: 710-717.

14. Schweitzer ME, Levine C, Mitchell DG, Gannon FH, Gomella LG. Bull's-eyes and halos: useful MR discriminators of osseous metastases. Radiology 1993; 188: 249-252.

15. Yamaguchi T. Intertrabecular vertebral metastases: metastases only detectable on MR imaging. Semin Musculoskelet Radiol 2001; 5: 171-175.

16. Taoka T, Mayr NA, Lee HJ, et al. Factors influencing visualization of vertebral metastases on MR imaging versus bone scintigraphy. Am J Roentgenol 2001; 176: 1525-1530.

17. Khurana JS, Rosenthal DI, Rosenberg AE, Mankin HJ. Skeletal metastases in liposarcoma detectable only by magnetic resonance imaging. Clin Orthop Relat Res 1989; 243: 204-207.

18. Islam A, Catovsky D, Galton DA. Histological study of bone marrow regeneration following chemotherapy for acute myeloid leukaemia and chronic granulocytic leukaemia in blast transformation. Br J Haematol 1980; 45: 535-540.

19. Sacks EL, Goris ML, Glatstein E, Gilbert E, Kaplan HS. Bone marrow regeneration following large field radiation: influence of volume, age, dose, and time. Cancer 1978; 42: 1057-1065. 
20. Sykes MP, Chu FC, Wilkerson WG. Local bone-marrow changes secondary to therapeutic irradiation. Radiology 1960; 75: 919-924.

21. Yankelevitz DF, Henschke CI, Knapp PH, Nisce L, Yi Y, Cahill P. Effect of radiation therapy on thoracic and lumbar bone marrow: evaluation with MR imaging. Am J Roentgenol 1991; 157: 87-92.

22. Blomlie V, Rofstad EK, Skjonsberg A, Tvera K, Lien HH. Female pelvic bone marrow: serial MR imaging before, during, and after radiation therapy. Radiology 1995; 194: 537-543.

23. Ramsey RG, Zacharias CE. MR imaging of the spine after radiation therapy: easily recognizable effects. Am J Roentgenol 1985; 144: 1131-1135.

24. Stevens SK, Moore SG, Kaplan ID. Early and late bone-marrow changes after irradiation: MR evaluation. Am J Roentgenol 1990; 154: 745-750.

25. Otake S, Mayr NA, Ueda T, Magnotta VA, Yuh WTC. Radiationinduced changes in MR signal intensity and contrast enhancement of lumbosacral vertebrae: do changes occur only inside the radiation therapy field? Radiology 2002; 222: 179-183.

26. Dalinka MK, Edeiken J, Finkelstein JB. Complications of radiation therapy: adult bone. Semin Roentgenol 1974; 9: 29-40.

27. Mitchell DG, Rao VM, Dalinka MK, et al. Femoral head avascular necrosis: correlation of MR imaging, radiographic staging, radionuclide imaging, and clinical findings. Radiology 1987; 162: 709-715.

28. Abe H, Nakamura M, Takahashi S, Maruoka S, Ogawa Y, Sakamoto K. Radiation-induced insufficiency fractures of the pelvis: evaluation with $99 \mathrm{mTc}$-methylene diphosphonate scintigraphy. Am J Roentgenol 1992; 158: 599-602.

29. Blomlie V, Lien HH, Iversen T, Winderen M, Tvera K. Radiationinduced insufficiency fractures of the sacrum: evaluation with MR imaging. Radiology 1993; 188: 241-244.

30. Mammone JF, Schweitzer ME. MRI of occult sacral insufficiency fractures following radiotherapy. Skeletal Radiol 1995; 24: 101-104.

31. Libshitz HI, Cohen MA. Radiation-induced osteochondromas. Radiology 1982; 142: 643-647.

32. Weatherby RP, Dahlin DC, Ivins JC. Postradiation sarcoma of bone: review of 78 Mayo Clinic cases. Mayo Clin Proc 1981; 56: 294-306.

33. Shaheen M, Deheshi BM, Riad S, et al. Prognosis of radiationinduced bone sarcoma is similar to primary osteosarcoma. Clin Orthop Relat Res 2006; 450: 76-81.

34. Kuttesch JF, Wexler LH, Marcus RB, et al. Second malignancies after Ewing's sarcoma: radiation dose-dependency of secondary sarcomas. J Clin Oncol 1996; 14: 2818-2825.

35. Inoue YZ, Frassica FJ, Sim FH, Unni KK, Petersen IA, McLeod RA. Clinicopathologic features and treatment of postirradiation sarcoma of bone and soft tissue. J Surg Oncol 2000; 75: 42-50.

36. Altehoefer $\mathrm{C}$, Laubenberger J, Lange $\mathrm{W}$, et al. Prospective evaluation of bone marrow signal changes on magnetic resonance tomography during high-dose chemotherapy and peripheral blood stem cell transplantation in patients with breast cancer. Invest Radiol 1997; 32: 613-620.

37. Schwartz AM, Leonidas JC. Methotrexate osteopathy. Skeletal Radiol 1984; 11: 13-16.

38. Hirbe A, Morgan EA, Uluckan O, Weilbaecher K. Skeletal complications of breast cancer therapies. Clin Cancer Res 2006; 12: $6309 \mathrm{~s}-6314 \mathrm{~s}$

39. Woo SB, Hellstein JW, Kalmar JR. Systematic review: bisphosphonates and osteonecrosis of the jaws. Ann Intern Med 2006; 144: 753-761.

40. Wood J, Bonjean K, Ruetz S, et al. Novel antiangiogenic effects of the bisphosphonate compound zoledronic acid. J Pharmacol Exp Ther 2002; 302: 1055-1061.

41. Fletcher BD, Wall JE, Hanna SL. Effect of hematopoietic growth factors on MR images of bone marrow in children undergoing chemotherapy. Radiology 1993; 189: 745-751.

42. Ryan SP, Weinberger E, White KS, et al. MR imaging of bone marrow in children with osteosarcoma: effect of granulocyte colony-stimulating factor. Am J Roentgenol 1995; 165: 915-920.

43. Hartman RP, Sundaram M, Okuno SH, Sim FH. Effect of granulocyte-stimulating factors on marrow of adult patients with musculoskeletal malignancies: incidence and MRI findings. Am J Roentgenol 2004; 183: 645-653.

44. Panicek DM, Schwartz LH. MR imaging of bone marrow in patients with musculoskeletal tumors. Sarcoma 1999; 3: 37-41.

45. Hanna SL, Fletcher BD, Fairclough DL, Jenkins JH, Le AH. Magnetic resonance imaging of disseminated bone marrow disease in patients treated for malignancy. Skeletal Radiol 1991; 20: 79-84.

46. Holscher HC, Bloem JL, Nooy MA, Taminiau AH, Eulderink F, Hermans $J$. The value of MR imaging in monitoring the effect of chemotherapy on bone sarcomas. Am J Roentgenol 1990; 154: 763-769.

47. Yasumoto M, Nonomura Y, Yoshimura R, et al. MR detection of iliac bone marrow involvement by malignant lymphoma with various MR sequences including diffusion-weighted echo-planar imaging. Skeletal Radiol 2002; 31: 263-269.

48. Gerard EL, Ferry JA, Amrein PC, et al. Compositional changes in vertebral bone marrow during treatment for acute leukemia: assessment with quantitative chemical shift imaging. Radiology 1992; 183: 39-46.

49. Vande Berg BC, Michaux L, Scheiff JM, et al. Sequential quantitative MR analysis of bone marrow: differences during treatment of lymphoid versus myeloid leukemia. Radiology 1996; 201: 519-523.

50. Dyke JP, Panicek DM, Healey JH, et al. Osteogenic and Ewing sarcomas: estimation of necrotic fraction during induction chemotherapy with dynamic contrast-enhanced MR imaging. Radiology 2003; 228: 271-278.

51. Lecouvet FE, Vande Berg BC, Michaux L, et al. Chronic lymphocytic leukemia: changes in bone marrow composition and distribution assessed with quantitative MRI. J Magn Reson Imaging 1998; 8: 733-739.

52. Le Bihan DJ. Differentiation of benign versus pathologic compression fractures with diffusion-weighted MR imaging: a closer step toward the "holy grail" of tissue characterization? [editorial]. Radiology 1998; 207: 305-307.

53. Wismer GL, Rosen BR, Buxton R, Stark DD, Brady TJ. Chemical shift imaging of bone marrow: preliminary experience. Am J Roentgenol 1985; 145: 1031-1037.

54. Rosen G, Caparros B, Huvos AG. Preoperative chemotherapy for osteogenic sarcoma: selection of postoperative adjuvant chemotherapy based on the response of the primary tumor to preoperative chemotherapy. Cancer 1982; 49: 1221-1230.

55. Meyers PA, Gorlick R, Heller G, et al. Intensification of preoperative chemotherapy for osteogenic sarcoma: results of the Memorial Sloan-Kettering (T12) protocol. J Clin Oncol 1998; 16: 2452-2458.

56. Lauenstein TC, Goehde SC, Herborn CU, et al. Three-dimensional volumetric interpolated breath-hold MR imaging for whole-body tumor staging in less than 15 minutes: a feasibility study. Am J Roentgenol 2002; 179: 445-449.

57. Johnston C, Brennan S, Ford S, Eustace S. Whole body MR imaging: applications in oncology. Eur J Surg Oncol 2006; 32: 239-246.

58. Ghanem N, Lohrmann C, Engelhardt M, et al. Whole-body MRI in the detection of bone marrow infiltration in patients with plasma cell neoplasms in comparison to the radiological skeletal survey. Eur Radiol 2006; 16: 1005-1014.

59. Mulligan MR, Badros AZ. PET/CT and MR imaging in myeloma. Skeletal Radiol 2007; 36: 5-16.

60. Lauenstein TC, Goehde SC, Herborn CU, et al. Whole-body MR imaging: evaluation of patients for metastases. Radiology 2004; 233 : 139-148.

61. Ghanem N, Altehoefer C, Kelly T, et al. Whole-body MRI in comparison to skeletal scintigraphy in detection of skeletal metastases in patients with solid tumors. In Vivo 2006; 20: 173-182. 